\title{
Synthesis Of New 3-Carboxamidine Iminocoumarins
}

\section{And Benzopyrano[2,3-d]pyrimidines}

Sihem Trichili, Myriam Kammoun, Hamida Turki-Guermazi,

Souhir Abid and Houcine Ammar

Laboratoire de Chimie Appliquée: Hétérocycles, Corps Gras et Polymères, Faculté des Sciences de Sfax, Université de Sfax, Route Soukra, BP1170, 3000 Sfax, Tunisie

\section{Supplementary Material}

Table of Contents

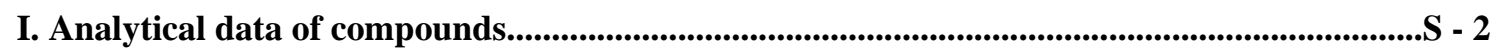

General procedure for the synthesis of 3-carboxamidine iminocoumarins 12-18................. - 2

General procedure for the synthesis of benzopyranopyrimidines 19-22 ….........................S - 5

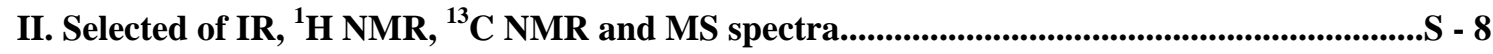




\section{Analytical data of compounds}

\section{General procedure for the synthesis of 3-carboxamidine iminocoumarins 12-18}

A mixture of 3-cyano-iminocoumarins (1-7) $(0.05 \mathrm{~mol})$ and cyanamide $(0.05 \mathrm{~mol})$, were stirred in $30 \mathrm{ml}$ of methanol. After total dissolution $0.05 \mathrm{~mol}$ of piperidine was added and the reaction mixture was stirred at room temperature during the time indicated in Table $\mathbf{1}$. After complete reaction, the simple 3-carboxamidine iminocoumarins obtained was separated by filtration and washed with methanol.

\section{$N$-cyano-2-imino-8-methoxy-2H-chromene-3-carboxamidine (12)}

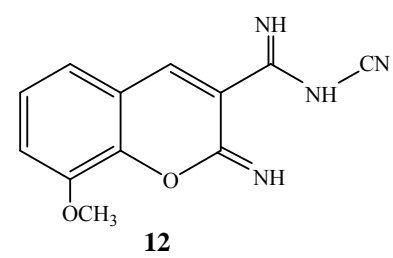

Yellow solid, IR Spectrum $\left(v: \mathrm{cm}^{-1}\right): 1597(\mathrm{C}=\mathrm{C}), 1620 / 1660(\mathrm{C}=\mathrm{N}), 2211(\mathrm{C} \equiv \mathrm{N})$, 3312/3342/3438 (NH). ${ }^{1} \mathrm{H}$ NMR Spectrum (300MHz, DMSO), ( $\delta:$ ppm, J: Hz): 3.89 (s, 3H, $\left.\mathrm{OCH}_{3}\right), 7.18-7.34\left(\mathrm{~m}, 3 \mathrm{H}, \mathrm{H}_{5}, \mathrm{H}_{6}, \mathrm{H}_{7}\right), 8.52\left(\mathrm{~s}, 1 \mathrm{H}, \mathrm{H}_{4}\right), 9.04(\mathrm{~s}, 1 \mathrm{H}, \mathrm{NH}), 9.20(\mathrm{~s}, 1 \mathrm{H}, \mathrm{NH})$, $10.88(\mathrm{~s}, 1 \mathrm{H}, \mathrm{NH})$. Calculated for $\mathrm{C}_{12} \mathrm{H}_{10} \mathrm{~N}_{4} \mathrm{O}_{2}: \% \mathrm{C} 59.50, \% \mathrm{H} 4.16, \% \mathrm{~N}$ 23.13. Found: $\% \mathrm{C}$ $59.43, \% \mathrm{H} 4.21, \% \mathrm{~N} 23.17$. 


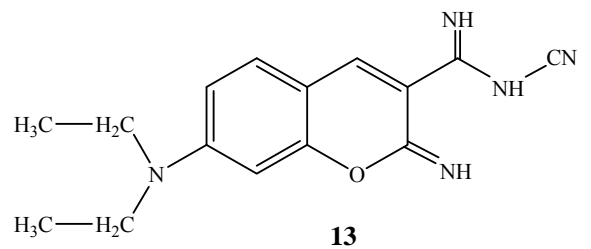

Orange-brown solid, IR Spectrum $\left(v\right.$ : $\left.\mathrm{cm}^{-1}\right): 1582(\mathrm{C}=\mathrm{C}), 1623 / 1657(\mathrm{C}=\mathrm{N}), 2213(\mathrm{C} \equiv \mathrm{N})$, 3311/3332/ 3344 (NH). ${ }^{1} \mathrm{H}$ NMR Spectrum (300 MHz, DMSO), ( $\delta:$ ppm, J: Hz): 1.10 (t, ${ }^{3} J=$ $\left.7.2 \mathrm{~Hz}, 6 \mathrm{H}, \mathrm{CH}_{3}\right), \delta=3.43\left(\mathrm{q},{ }^{3} J=7.2 \mathrm{~Hz}, 4 \mathrm{H}, \mathrm{CH}_{2}\right), 6.26\left(\mathrm{~s}, 1 \mathrm{H}, \mathrm{H}_{8}\right), 6.63\left(\mathrm{~d},{ }^{3} J=8.7 \mathrm{~Hz}\right.$, $\left.1 \mathrm{H}, \mathrm{H}_{6}\right), 7.34\left(\mathrm{~d},{ }^{3} J=8.7 \mathrm{~Hz}, 1 \mathrm{H}, \mathrm{H}_{5}\right), 8.16\left(\mathrm{~s}, 1 \mathrm{H}, \mathrm{H}_{4}\right) 8.43(\mathrm{~s}, 1 \mathrm{H}, \mathrm{NH}), 8.62(\mathrm{~s}, 1 \mathrm{H}, \mathrm{NH})$, $9.86(\mathrm{~s}, 1 \mathrm{H}, \mathrm{NH})$. Calculated for $\mathrm{C}_{15} \mathrm{H}_{17} \mathrm{~N}_{5} \mathrm{O}: \% \mathrm{C} 63.60, \% \mathrm{H} 6.00, \% \mathrm{~N}$ 24.72.Found: $\% \mathrm{C}$ $63.67, \% \mathrm{H} 5.92, \% \mathrm{~N} 24.67$.

N-cyano-2-imino-7-methoxy-2H-chromene-3-carboxamidine (14)<smiles>CNC(=N)c1cc2ccc(OC)cc2oc1=N</smiles>

Yellow solid, IR Spectrum $\left(v: \mathrm{cm}^{-1}\right): 1584(\mathrm{C}=\mathrm{C}), 1621 / 1662(\mathrm{C}=\mathrm{N}), 2220(\mathrm{C} \equiv \mathrm{N})$, 3300/3324/3392 (NH). ${ }^{1} \mathrm{H}$ NMR Spectrum (300MHz, DMSO), ( $\delta:$ ppm, J: Hz): 3.84 (s, 3H, $\left.\mathrm{OCH}_{3}\right), 6.74\left(\mathrm{~s}, 1 \mathrm{H}, \mathrm{H}_{8}\right), 6.92\left(\mathrm{~d},{ }^{3} J=8,4 \mathrm{~Hz}, 1 \mathrm{H}, \mathrm{H}_{6}\right), 7.64\left(\mathrm{~d},{ }^{3} J=8,4 \mathrm{~Hz}, 1 \mathrm{H}, \mathrm{H}_{5}\right)$, $8.58\left(\mathrm{~s}, 1 \mathrm{H}, \mathrm{H}_{4}\right) .8 .83(\mathrm{~s}, 1 \mathrm{H}, \mathrm{NH}), 9.07(\mathrm{~s}, 1 \mathrm{H}, \mathrm{NH}), 10.60(\mathrm{~s}, 1 \mathrm{H}, \mathrm{NH})$. Calculated for $\mathrm{C}_{12} \mathrm{H}_{10} \mathrm{~N}_{4} \mathrm{O}_{2}$ : \% $59.50, \% \mathrm{H} 4.16, \% \mathrm{~N}$ 23.13. Found: \%C 59.57, \%H 4.07, \%N 23.09. 
$N$-cyano-2-imino-6-methoxy-2H-chromene-3-carboxamidine (15)

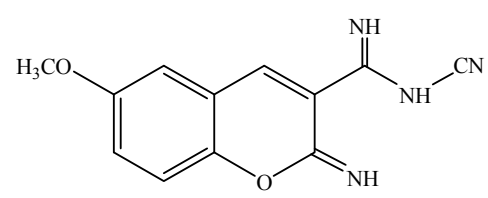

15

Orange solid, IR Spectrum $\left(v: \mathrm{cm}^{-1}\right): 1595(\mathrm{C}=\mathrm{C}), 1618 / 1658(\mathrm{C}=\mathrm{N}), 2218(\mathrm{C} \equiv \mathrm{N})$, 3304/3329/3348 (NH). ${ }^{1} \mathrm{H}$ NMR Spectrum (300MHz, DMSO), ( $\delta:$ ppm, J: Hz): 3.78 (s, 3H, $\left.\mathrm{OCH}_{3}\right), 7.16\left(\mathrm{~d},{ }^{3} \mathrm{~J}=8.7 \mathrm{~Hz}, 1 \mathrm{H}, \mathrm{H}_{8}\right), 7.24\left(\mathrm{~d},{ }^{3} \mathrm{~J}=2.1 \mathrm{~Hz}, 1 \mathrm{H}, \mathrm{H}_{5}\right), 7.32\left(\mathrm{dd},{ }^{3} J=2.1 \mathrm{~Hz},{ }^{3} \mathrm{~J}=\right.$ $\left.8.7 \mathrm{~Hz}, 1 \mathrm{H}, \mathrm{H}_{7}\right), 8.38\left(\mathrm{~s}, 1 \mathrm{H}, \mathrm{H}_{4}\right), 8.92(\mathrm{~s}, 1 \mathrm{H}, \mathrm{NH}), 9.13$ (s, 1H, NH), 10.54 (s, 1H, NH). Calculated for $\mathrm{C}_{12} \mathrm{H}_{10} \mathrm{~N}_{4} \mathrm{O}_{2}$ : \%C 59.50, \%H 4.16, \%N 23.13. Found: \%C 59.45, \%H 4.28, $\%$ N 23.07.

6-chloro- $N$-cyano-2-imino-2H-chromene-3-carboxamidine (16)

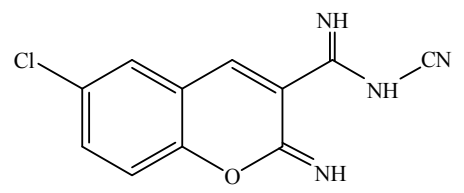

16

Red-brown solid, IR Spectrum $\left(v: \mathrm{cm}^{-1}\right): 1583(\mathrm{C}=\mathrm{C}), 1628 / 1668 \quad(\mathrm{C}=\mathrm{N}), 2220(\mathrm{C} \equiv \mathrm{N})$, 3307/3322/3341 (NH). ${ }^{1} \mathrm{H}$ NMR Spectrum (300MHz, DMSO), ( $\delta:$ ppm, J: Hz): 7.42 (d, ${ }^{3} J=$ $\left.6.9 \mathrm{~Hz}, 1 \mathrm{H}, \mathrm{H}_{8}\right), 7.64\left(\mathrm{dd},{ }^{4} \mathrm{~J}=1.8 \mathrm{~Hz},{ }^{3} \mathrm{~J}=6.9 \mathrm{~Hz}, 1 \mathrm{H}, \mathrm{H}_{7}\right), 7.73\left(\mathrm{~d},{ }^{4} \mathrm{~J}=1.8 \mathrm{~Hz}, 1 \mathrm{H}, \mathrm{H}_{5}\right)$, $8.34\left(\mathrm{~s}, 1 \mathrm{H}, \mathrm{H}_{4}\right), 9.05(\mathrm{~s}, 1 \mathrm{H}, \mathrm{NH}), 9.23(\mathrm{~s}, 1 \mathrm{H}, \mathrm{NH}), 10.89(\mathrm{~s}, 1 \mathrm{H}, \mathrm{NH})$. Calculated for $\mathrm{C}_{11} \mathrm{H}_{7} \mathrm{ClN}_{4} \mathrm{O}$ : \%C 53.55, \%H 2.84, \%N 22.71. Found: \%C 53.61, \%H 2.80, \%N 22.78. 
N-cyano-7-hydroxy-2-imino-2H-chromene-3-carboxamidine (17)

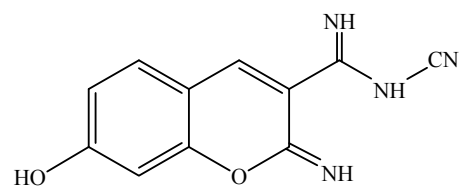

17

Yellow solid, IR Spectrum $\left(v: \mathrm{cm}^{-1}\right): 1586(\mathrm{C}=\mathrm{C}), 1632 / 1672 \quad(\mathrm{C}=\mathrm{N}), 2211(\mathrm{C} \equiv \mathrm{N})$, 3312/3332 (NH), $3428(\mathrm{OH}) .{ }^{1} \mathrm{H}$ NMR Spectrum (300MHz, DMSO), ( $\delta:$ ppm, J: Hz): 6.56 (s, $\left.1 \mathrm{H}, \mathrm{H}_{8}\right), 6.69\left(\mathrm{~d},{ }^{3} J=7.2 \mathrm{~Hz}, 1 \mathrm{H}, \mathrm{H}_{6}\right), 7.43\left(\mathrm{~d},{ }^{3} J=7.2 \mathrm{~Hz}, 1 \mathrm{H}, \mathrm{H}_{5}\right), 8.29\left(\mathrm{~s}, 1 \mathrm{H}, \mathrm{H}_{4}\right), 8.64(\mathrm{~s}$, $1 \mathrm{H}, \mathrm{NH}), 8.83(\mathrm{~s}, 1 \mathrm{H}, \mathrm{NH}), 9.34(\mathrm{~s}, 1 \mathrm{H}, \mathrm{OH}) 9.74(\mathrm{~s}, 1 \mathrm{H}, \mathrm{NH})$. Calculated for $\mathrm{C}_{11} \mathrm{H}_{8} \mathrm{~N}_{4} \mathrm{O}_{2}$ : \%C 57.89, \%H 3.53, \%N 24.55. Found: \%C 57.82, \%H 3.60, \%N 24.45.

N-cyano-2-imino-5,6-phenyl-2H-chromene-3-carboxamidine (18)

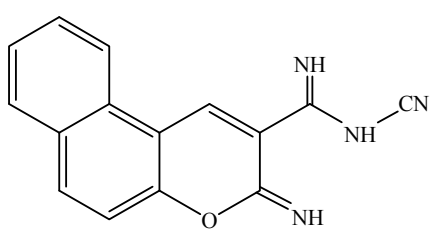

18

Orange-brown solid, IR Spectrum $\left(v: \mathrm{cm}^{-1}\right)$ : $1596(\mathrm{C}=\mathrm{C}), 1625 / 1671(\mathrm{C}=\mathrm{N}), 2219(\mathrm{C} \equiv \mathrm{N})$, 3310/3334/3378 (NH). ${ }^{1} \mathrm{H}$ NMR Spectrum (300 MHz, DMSO), ( $\delta:$ ppm, J: Hz): 7.58 (d, $\left.{ }^{3} J=8.2 \mathrm{~Hz}, 1 \mathrm{H}, \mathrm{H}_{8}\right), 7.72-8.62\left(\mathrm{~m}, 5 \mathrm{H}, \mathrm{H}_{7}, \mathrm{H}_{\mathrm{Ar}}\right), 8.98\left(\mathrm{~s}, 1 \mathrm{H}, \mathrm{H}_{4}\right), 9.13(\mathrm{~s}, 1 \mathrm{H}, \mathrm{NH}), 9.25(\mathrm{~s}$, $1 \mathrm{H}, \mathrm{NH}), 10.80$ (s, 1H, NH). Calculated for $\mathrm{C}_{15} \mathrm{H}_{10} \mathrm{~N}_{4} \mathrm{O}: \% \mathrm{C} 68.70, \% \mathrm{H} 3.82, \% \mathrm{~N} 21.37$. Found: $\% \mathrm{C} 68.95, \% \mathrm{H} 3.75, \% \mathrm{~N} 21.29$.

General procedure for the synthesis of benzopyrano[2,3-d]pyrimidines 19-22 
A mixture of 3-cyano-N-ethoxycarbonyl iminocoumarins 8-11 $(0.05 \mathrm{~mol})$ and cyanamide $(0.05 \mathrm{~mol})$ in $30 \mathrm{ml}$ of absolute methanol was refluxed during the time indicated in Table 2. The precipitate was filtered and washed with methanol.

\section{$N$-cyano-8-diethylamino-4-imino-2-oxo-2H-Benzopyrano[2,3-d]pyrimidine (19)}<smiles>CCN(CC)c1ccc2cc3c(=N)n(C)c(=O)nc-3oc2c1</smiles>

Pink solid, IR Spectrum $\left(v: \mathrm{cm}^{-1}\right)$ : $1531(\mathrm{C}=\mathrm{C}), 1618 / 1647(\mathrm{C}=\mathrm{N}), 1709(\mathrm{C}=\mathrm{O}), 2189(\mathrm{C} \equiv \mathrm{N})$, $3130(\mathrm{NH}) .{ }^{1} \mathrm{H}$ NMR Spectrum (300MHz, DMSO), ( $\left.\delta: \mathrm{ppm}, \mathrm{J}: \mathrm{Hz}\right): 1.20\left(\mathrm{t},{ }^{3} \mathrm{~J}=6 \mathrm{~Hz}, 6 \mathrm{H}\right.$, $\left.\mathrm{CH}_{3}\right), 3.61\left(\mathrm{q},{ }^{3} \mathrm{~J}=6 \mathrm{~Hz}, 4 \mathrm{H}, \mathrm{CH}_{2}\right), 6.86\left(\mathrm{~d},{ }^{4} \mathrm{~J}=3 \mathrm{~Hz}, 1 \mathrm{H}, \mathrm{H}_{9}\right), 7.03\left(\mathrm{dd},{ }^{4} \mathrm{~J}=3 \mathrm{~Hz},{ }^{3} J=9.3\right.$ $\left.\mathrm{Hz}, 1 \mathrm{H}, \mathrm{H}_{7}\right), 7.79\left(\mathrm{~d},{ }^{3} \mathrm{~J}=9 \mathrm{~Hz}, 1 \mathrm{H}, \mathrm{H}_{6}\right), 8.86\left(\mathrm{~s}, 1 \mathrm{H}, \mathrm{H}_{5}\right), 11.58(\mathrm{~s}, 1 \mathrm{H}, \mathrm{NH}) .{ }^{13} \mathrm{C}$ NMR Spectrum (75MHz, DMSO), ( $\delta:$ ppm): 12.88, 45.59, 97.16, 102.72, 111.17, 113.39, 115.31, 133.92, 143.25, 155.54, 155.87, 157.88, 160.50, 167.36. Calculated for $\mathrm{C}_{16} \mathrm{H}_{15} \mathrm{~N}_{5} \mathrm{O}_{2}: \% \mathrm{C}$ $62.13, \% \mathrm{H} 4.85, \% \mathrm{~N} 22.65$. Found: $\% \mathrm{C} 62.19, \% \mathrm{H} 4.96, \% \mathrm{~N} 22.55$. The mass spectrum was $\mathrm{m} / \mathrm{z} 310.4\left[(\mathrm{M}+\mathrm{H})^{+}\right]$.

$N$-cyano-8- methoxy-4-imino-2-oxo-2H-Benzopyrano[2,3-d]pyrimidine (20)<smiles>COc1ccc2cc3c(=N)n(C#N)c(=O)nc-3oc2c1</smiles> 
Pink solid, IR Spectrum ( $v$ : $\left.\mathrm{cm}^{-1}\right): 1530(C=C), 1617 / 1643(C=N), 1703(C=O), 2211(C \equiv N)$, 3223 (NH). ${ }^{1} \mathrm{H}$ NMR Spectrum (300MHz, DMSO), ( $\delta:$ ppm, J: Hz): 3.32 (s, 3H, $\left.\mathrm{OCH}_{3}\right), 6.70$ $\left(\mathrm{d},{ }^{4} \mathrm{~J}=2.4 \mathrm{~Hz}, 1 \mathrm{H}, \mathrm{H}_{9}\right), 6.94\left(\mathrm{dd},{ }^{4} \mathrm{~J}=2.4 \mathrm{~Hz},{ }^{3} \mathrm{~J}=9.3 \mathrm{~Hz}, 1 \mathrm{H}, \mathrm{H}_{7}\right), 7.74\left(\mathrm{~d},{ }^{3} \mathrm{~J}=9.3 \mathrm{~Hz}, 1 \mathrm{H}\right.$, $\left.\mathrm{H}_{6}\right), 8.62\left(\mathrm{~s}, 1 \mathrm{H}, \mathrm{H}_{5}\right), 11.13(\mathrm{~s}, 1 \mathrm{H}, \mathrm{NH}) .{ }^{13} \mathrm{C}$ NMR Spectrum (75MHz, DMSO), ( $\left.\delta: \mathrm{ppm}\right)$ : $55.90,98.13,104.20,109.23,110.89,117.12,126.92,132.25,158.24,160.17,160.88,161.50$, 166.86. Calculated for $\mathrm{C}_{13} \mathrm{H}_{8} \mathrm{~N}_{4} \mathrm{O}_{3}$ : \% $58.21, \% \mathrm{H} 3.01, \% \mathrm{~N} 20.89$. Found: $\% \mathrm{C} 58.14, \% \mathrm{H}$ $3.09, \% \mathrm{~N} 20.84$.

$N$-cyano-7- methoxy-4-imino-2-oxo-2H-Benzopyrano[2,3-d]pyrimidine (21)

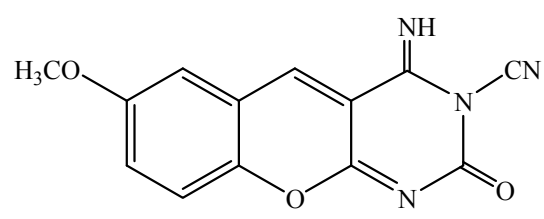

21

Pink solid, IR Spectrum (v: $\left.\mathrm{cm}^{-1}\right)$ : $1532(\mathrm{C}=\mathrm{C}), 1623 / 1638(\mathrm{C}=\mathrm{N}), 1708(\mathrm{C}=\mathrm{O}), 2179(\mathrm{C} \equiv \mathrm{N})$, 3200 (NH). ${ }^{1} \mathrm{H}$ NMR Spectrum (300MHz, DMSO), ( $\delta:$ ppm, J: Hz): 3.58 (s, 3H, $\left.\mathrm{OCH}_{3}\right), 6.82$ $\left(\mathrm{d},{ }^{3} J=7.4 \mathrm{~Hz}, 1 \mathrm{H}, \mathrm{H}_{9}\right), 7.08\left(\mathrm{~s}, 1 \mathrm{H}, \mathrm{H}_{6}\right), 7.72\left(\mathrm{~d},{ }^{3} J=7.4 \mathrm{~Hz}, 1 \mathrm{H}, \mathrm{H}_{8}\right), 8.62\left(\mathrm{~s}, 1 \mathrm{H}, \mathrm{H}_{5}\right)$, 10.98 (s, 1H, NH). ${ }^{13} \mathrm{C}$ NMR Spectrum (75MHz, DMSO), ( $\left.\delta: \mathrm{ppm}\right): 55.30,104.92,109.22$, $111.64,114.13,115.17,115.31,139.05,155.29,156.11,159.11,161.23,166.69$. Calculated for $\mathrm{C}_{13} \mathrm{H}_{8} \mathrm{~N}_{4} \mathrm{O}_{3}: \% \mathrm{C} 58.21, \% \mathrm{H} 3.01, \% \mathrm{~N} 20.89$. Found: \%C 58.28, \%H 3.07, \%N 20.79.

$N$-cyano-9- methoxy-4-imino-2-oxo-2H-Benzopyrano[2,3-d]pyrimidine (22)<smiles>COc1cccc2cc3c(=N)n(C#N)c(=O)nc-3oc12</smiles> 
Pink solid, IR Spectrum (v: $\left.\mathrm{cm}^{-1}\right): 1528(\mathrm{C}=\mathrm{C}), 1613 / 1645(\mathrm{C}=\mathrm{N}), 1693(\mathrm{C}=\mathrm{O}), 2189(\mathrm{C} \equiv \mathrm{N})$, $3134(\mathrm{NH}) .{ }^{1} \mathrm{H}$ NMR Spectrum (300MHz, DMSO), ( $\left.\delta: \mathrm{ppm}, \mathrm{J}: \mathrm{Hz}\right): 3.82\left(\mathrm{~s}, 3 \mathrm{H}, \mathrm{OCH}_{3}\right)$, 7.68-7.83 (m, 3H, $\left.\mathrm{H}_{6}, \mathrm{H}_{7}, \mathrm{H}_{8}\right), 8.68\left(\mathrm{~s}, 1 \mathrm{H}, \mathrm{H}_{5}\right), 11.62(\mathrm{~s}, 1 \mathrm{H}, \mathrm{NH}) .{ }^{13} \mathrm{C} \mathrm{NMR}$ Spectrum(75MHz, DMSO), (ס: ppm): 59.98, 102.22, 112.27, 115.21, 118.23, 122.11, 125.69, $142.76,149.09,152.56,158.01,159.55,165.89$. Calculated for $\mathrm{C}_{13} \mathrm{H}_{8} \mathrm{~N}_{4} \mathrm{O}_{3}: \% \mathrm{C} 58.21, \% \mathrm{H}$ 3.01, \%N 20.89. Found: \%C 58.15, \%H 2.91, \%N 20.96. 
II. Selected of IR, ${ }^{1} \mathrm{H}$ NMR, ${ }^{13} \mathrm{C}$ NMR and MS spectra 


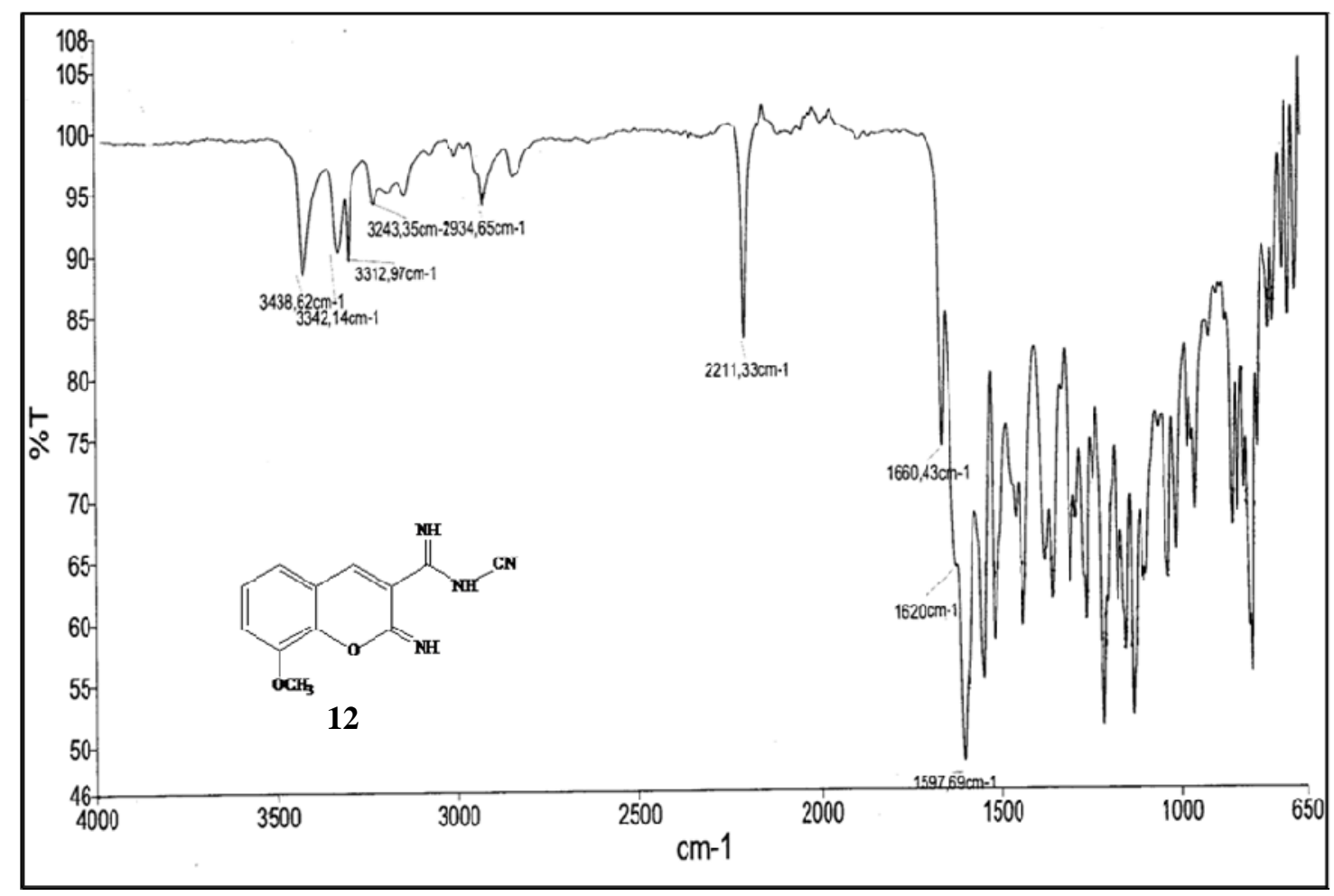




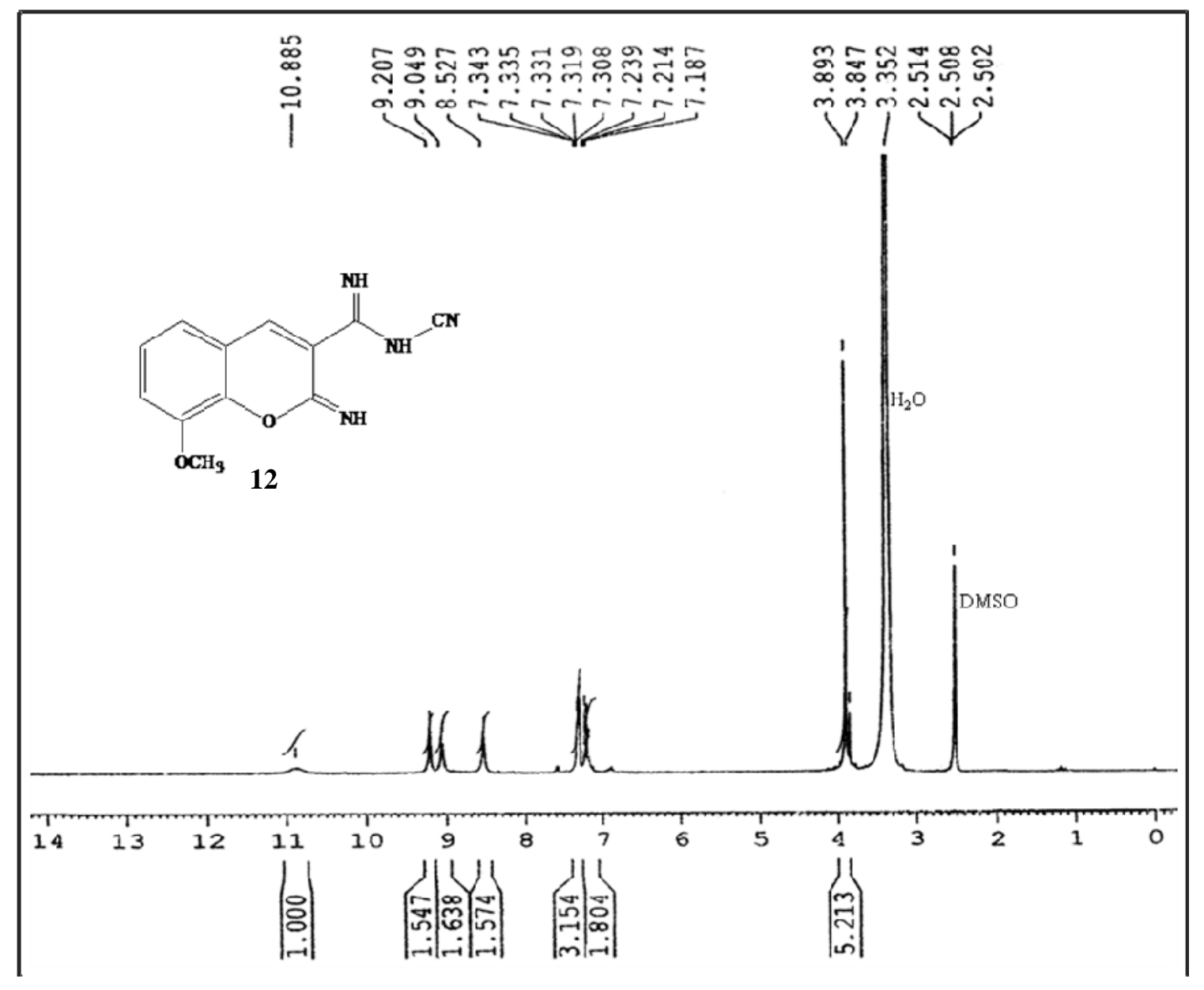




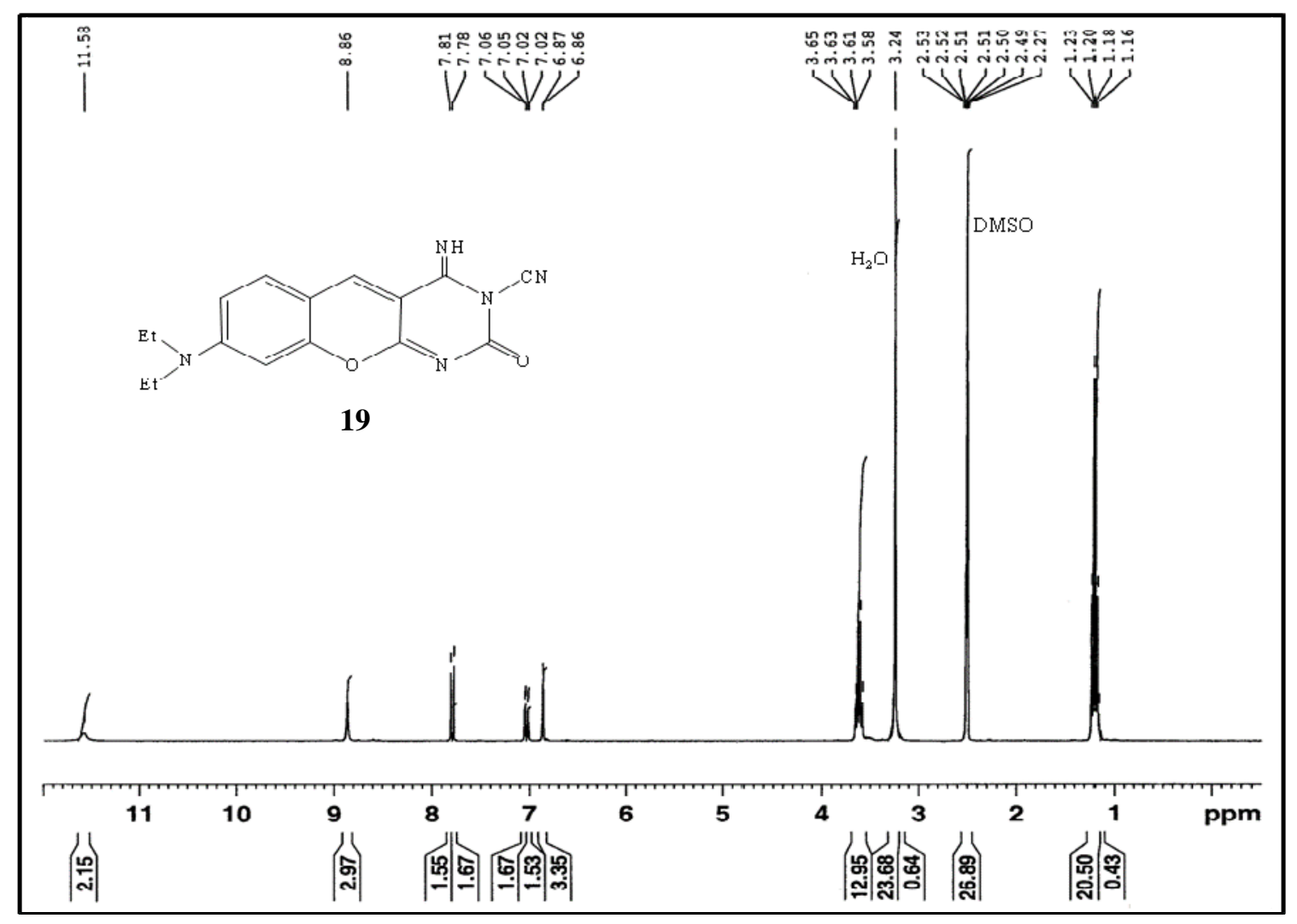




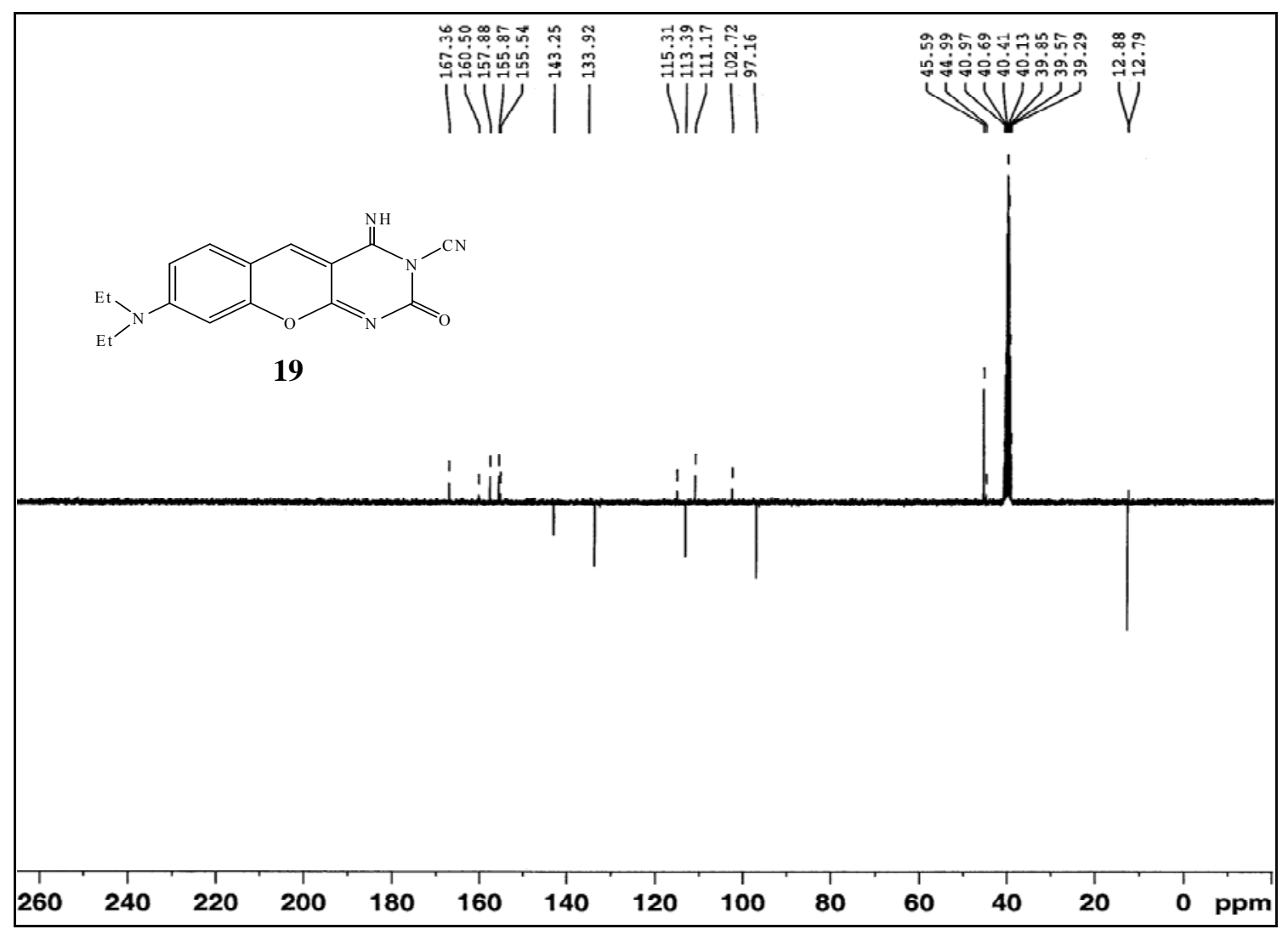




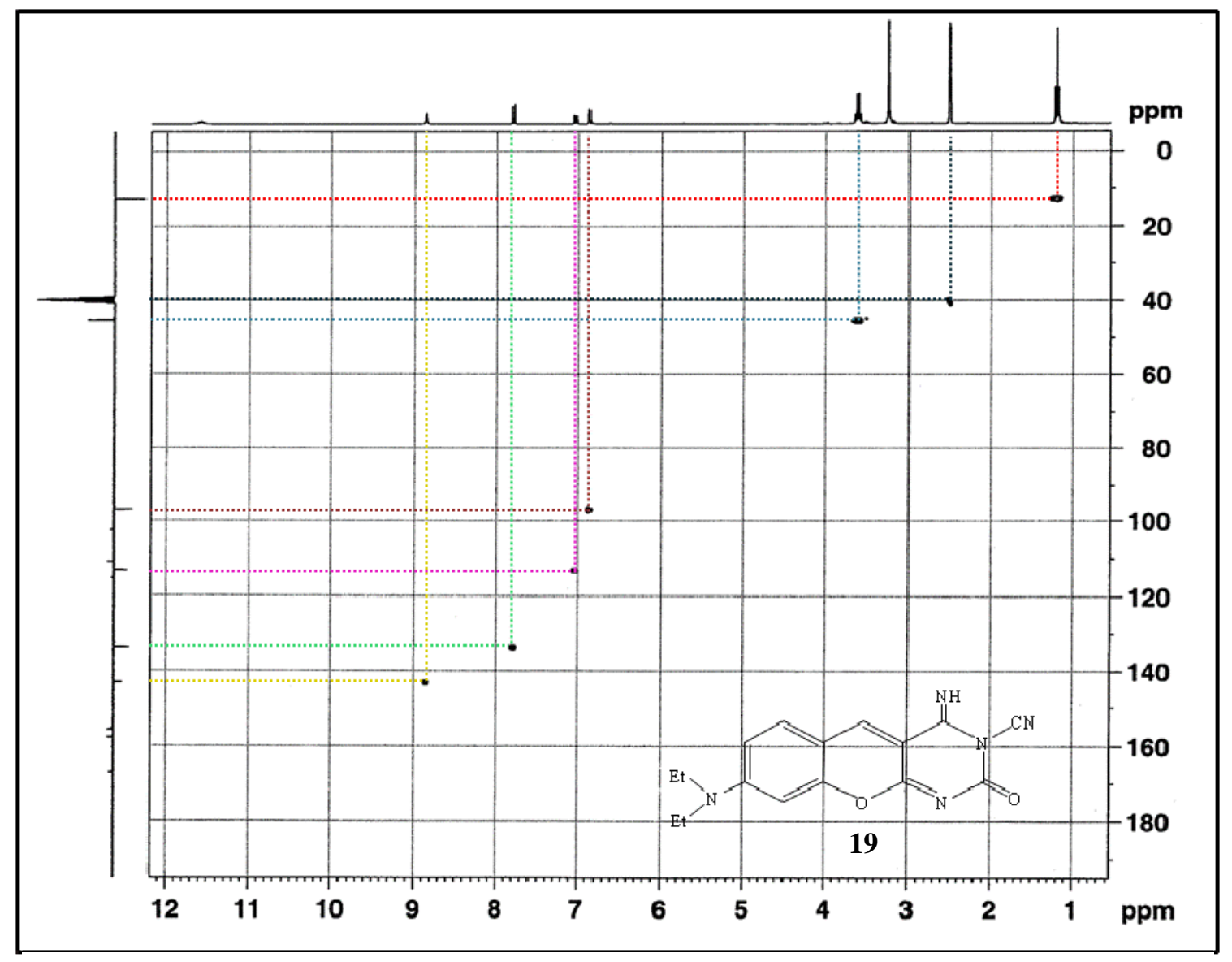



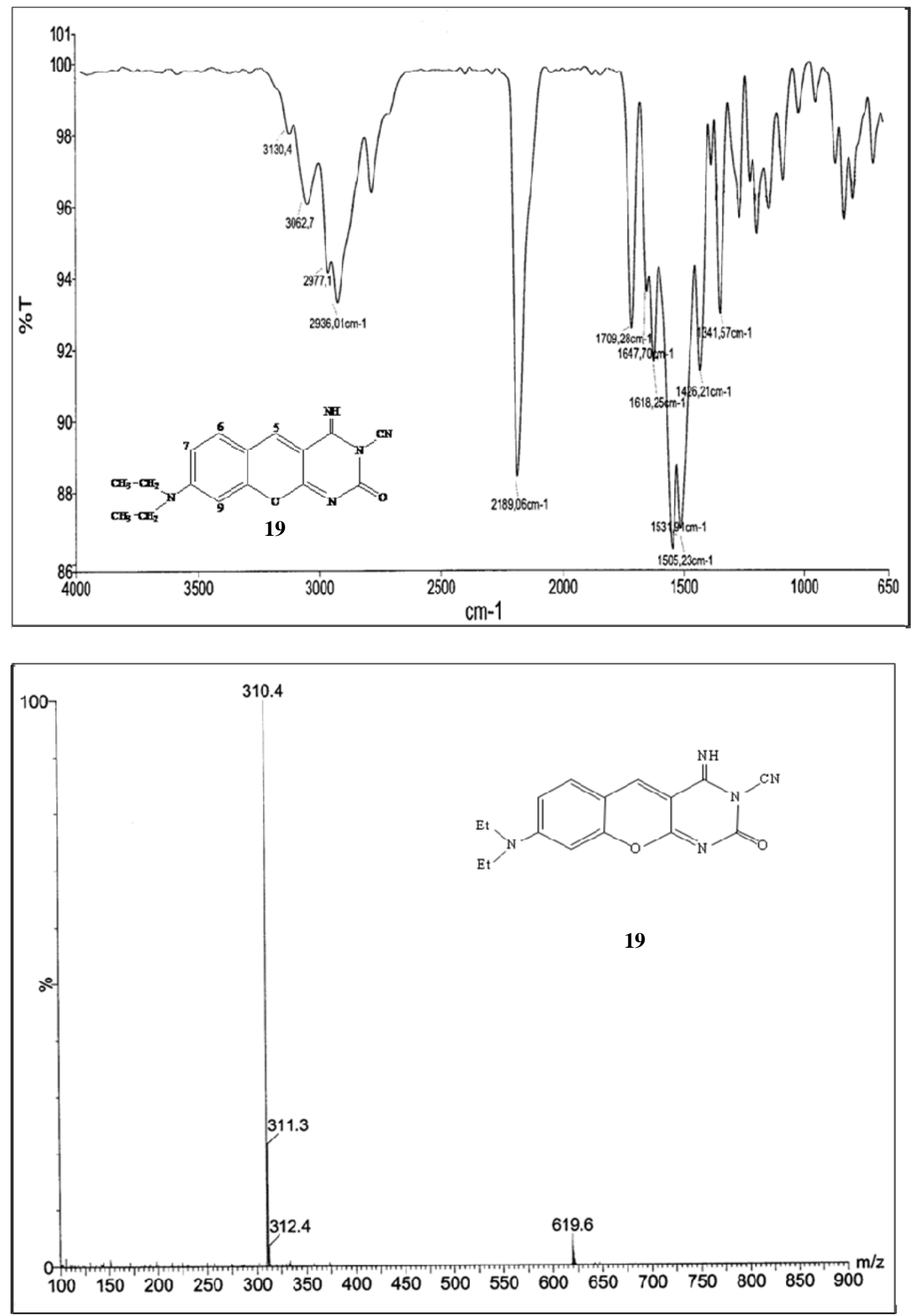
